\title{
XXII. On the refraction and polarization of heat
}

\author{
James D. Forbes Esq. F.R.SS. L. E.
}

To cite this article: James D. Forbes Esq. F.R.SS. L. E. (1835) XXII. On the refraction and polarization of heat, Philosophical Magazine Series 3, 6:32, 134-142, DOI: 10.1080/14786443508648549

To link to this article: http://dx.doi.org/10.1080/14786443508648549

曲 Published online: 01 Jun 2009.

Submit your article to this journal

Џ Article views: 5

Q View related articles $\sqsubset$ 


\section{Prof. Forbes on the Refraction and Polarization of Heat.}

The change of colour which is exhibited by looking through the mineral in day-and in candle-light, arises from two causes : 1 st, from there being an excess of red and a defect of blue rays in the light of a candle compared with the light of day; and 2ndly, from the substance employed having a greater disposition to transmit one kind of rays in preference to another, or, what is the same thing, being more transparent for one kind of rays than for another kind, when their intensity is the same.

In the present mineral its colour is green; but when we analyse it with the prism we find that the green is a compound colour consisting of red and green, the green predominating greatly in daylight: but in candlelight the colour is a pink red, because the greater quantity of red in this light and the smaller quantity of blue and green, gives the red colour a decided predominance over the green, so as to make the compound colour pink red.

There are several crystals, natural and artificial, and various solutions in which this change of colour is beautifully seen. It is particularly visible in the green juices of plants, which are green in daylight, and of a blood red colour in the light of a candle.

In the mineral under our consideration Mr. Nordenskiold found traces of the oxide of chromium, to which he attributes its colour. That this is the colouring matter, and that the action of this metal is the cause of its peculiar property in reference to light, may be inferred from the fact that the very same property is possessed by the triple oxalate of chromium and potash, and also by the sulphate of ammonia and chromium, whether these salts are used in the solid state or in a state of solution.

XXII. On the Refraction and Polarization of Heat. By James D. Forbes, Esq., F.R.SS. L.\& E., Professor of Natural Philosophy in the University of Edinburgh.*

$\oint 1$. Some Miscellaneous Experiments with the ThermoMultiplier. \$2. On the Polarization of Heat by Tourmaline. \$ 3. On the Polarization of Heat by Refraction and Reflection. $\$ 4$. On the Depolarization and Double Refraction of Heat.

1. THE experiments to be detailed in this paper, which chiefly go to establish properties of heat wholly unlooked for, or only suspected to exist, having been made en-

* Communicated by the Author; having been read before the Royal Society of Edinburgh on the 5th and 19th of January 1835. 
tirely by means of an instrument of great delicacy-the thermo-multiplier of MM. Nobili and Melloni,-I shall premise some account of its application to the investigation of some more familiar modes of action.

\section{$\S 1$. Miscellaneous Experiments.}

2. We could hardly quote a stronger proof of the rapid and unexpected advances which enlarged theory may produce in practice, than by referring to the employment of thermoelectric action, discovered a few years since by Seebeck, to the measurement of heat, with a degree of accuracy and facility. which, perhaps, no thermometer has ever attained. Such is the principle of the thermo-multiplier of Nobili and Melloni. It is well known, that when two metals (and especially bismuth and antimony) are soldered together, and the point of union heated, an electric current is established from the one metal to the other, which may be carried off by wires, and caused to act upon a delicate galvanometer or multiplier, the needle of which serves as an index; the galvanometer consisting, of course, of a magnetic needle, nearly freed from the influence of the earth's magnetism, and so connected with the wire which transmits the electricity, that the mutual influence of the magnetism and the electricity shall (by the law of (Ersted) be a maximum.

3. It will readily be conceived, that, if a series of alternating bars of bismuth and antimony be placed parallel to each other, and the extremities alternately soldered together, when all the extremities facing one way are heated (as by the radiant influence of a lamp), whilst the others remain at the temperature of the apartment, the effects produced in a single pair, such as we first supposed, will be produced at each junction, and that the intensity of the whole effect will be greater, just as in the voltaic pile. At one time it appeared doubtful how far electricity, of such small tension as is thus produced, could be so reinforced; but the instrument in question seems to prove the practicability of it. About thirty pairs are employed, and so delicately are they made, that the ends which exhibit one set of junctions are contained within a superficial area of four tenths of an inch square.

4. The wires, from the extremity of the first and last element (just as in the voltaic battery), convey the electricity to the multiplier, which consists of a flattened coil of silver-wire, covered with silk, the coils of the wire being parallel to the quiescent position of an astatic magnetic needle, which is perpendicular to the magnetic meridian. The deviations are measured in the usual manner, on a divided circle; upon 


\section{Prof. Forbes on the Refraction and Polarization of Heat.}

which, with practice, $\frac{1}{4}$ th of a degree may always be observed, and even minuter quantities occasionally estimated. These divisions are not necessarily proportional to the intensities of the currents which produce the corresponding deviations. The coils of wire, extending a long way on each side of zero, prevent the effect from diminishing so rapidly as if they were concentrated there; and M. Melloni has described, in his paper in the Annales de Chimie for May 1833, a simple and satisfactory method of estimating the relative values of degrees, at different points of the scale. He states, however, that, under $20^{\circ}$ of deviation, he found them quite uniform. In the following experiments, the deviations were generally under $15^{\circ}$, and in almost no case exceeded $20^{\circ}$. I have therefore assumed the forces to be as the deviations. Besides, no change of importance would take place from a deviation from this law by a small quantity.

5. It will be perceived in the experiments which are to be detailed, that the determination of all the inore important facts depend generally on whether one effect be greater or less than another, without much regard to their absolute amount. Now, the confidence which we can place in the uniformity of this instrument, or at least of the small changes capable of affecting it (since it is not liable like thermometers, and especially air-thermometers, to advance by starts,) is such, as to admit of almost indefinite subdivision, where the relations of small quantities are alone concerned. I conceived, therefore, that without impairing its sensibility by lengthening the galvanometer needle, we might advantageously magnify the divisions by optical means. This I proposed to do by observing the motions of the index by means of a small telescope, fixing in front of the object glass a lens whose focus is situated at the part of the scale desired to be magnified. It might also be easy, in order to compare larger quantities, to make this micrometrical system revolve so as to be always similarly placed as regards the needle, and thus avoid the effects of parallax, which at present require constant vigilance.

6. The method here indicated, I have put into practice with the greatest success in my later researches; one tenth of a degree becomes easily visible, and the constancy of the indications fully justifies this method of microscopic examination, which has enabled me to verify the most delicate deductions I had drawn from simple observation, and to obtain results which otherwise I must have been unable confidently to announce.

7. For the precautions to be employed in the use of the thermo-multiplier, I must refer to the first of M. Melloni's very original papers in the Annales de Chimie (for May 1833), 
but I may state, once for all, that when once habituated to the use of it, I have found it more simple, manageable, and comparable, than I could previously have imagined. Notwithstanding its delicacy and the promptitude of its action, a few precautions suffice to prevent any derangement from without. The only inconvenience which I experienced, was in the determination of the zero of the scale, which appears liable to some fluctuations, which may be considered as accidental. It rarely happened, however, that these affected the results of my experiments, because, as I have said, these were always confined to small variations of temperature (indicated by a deviation generally under $15^{\circ}$ on the scale) when such fluctuations did not appear; and the results produced by the same cause under the same circumstances were admirably constant, as well as the position of the zero point.

8. There is one circumstance which gives a degree of delicacy to the indications of the thermo-multiplier, when we wish to ascertain very minute differences of effect, which no other thermometric instrument possesses. When we wish to ascertain the existence, not the measure, of some cause of heat or cold, if we watch the needle of the multiplier at the instant at which the change of circumstances intended to produce the effect takes place, we shall perceive, in the instantaneous effect on the needle, an evidence of a far more decisive character than the merely statical deviation (at which, after several oscillations, it is finally to settle) could afford. Not only does the acquired velocity carry it through double the space due to the statical effect; but I have observed that the action of the thermo-electric pile so far resembles that of the voltaic, that we appear to have an excess of effect at the first moment of action, which gives a greater deviation than can be afterwards obtained*. It is therefore to be recollected, that, in speaking confidently of effects, which, statically speaking, are exceedingly small, the experimentalist has a species of evidence far stronger than the mere numerical expression of the deviation of the needle, but the degree of which must be taken on the

- This remarkable effect, which may be described as an increase of tension by confinement, seems generally to exist where the conductors of imponderable agents oppose considerable resistance to their passage. It is familiar in voltaic electricity, and I have often observed it in magnetic electricity. It is similar to the action which $I$ have attempted to demonstrate in the passage of heat frorn good to bad conductors (see Lond. and Edinb. Phil. Mag., vol. iv., p. 15, et seq.), where we have the full advantage of the dynamical effect; whilst the existence of statical tension in heat seems likewise to be proved (as we might have anticipated) by the beautiful experiment described by Professor Powell in the Philosophical Transactions for 1834 , and noticed in the last number of this Journal, p. 58.

Third Series. Vol. 6. No. :2. Feb. 1835. 
138 Prof. Forbes on the Refraction and Polarization of Heat.

faith of his veracity. Thus I have obtained repeated differences, not exceeding half or even $a$ quarter of $a$ degree of the multiplier (observed without a telescope), which, by the promptitude with which the needle was repelled or attracted at the instant that the change of circumstances to be considered was effected, left as little doubt in my mind as if the numerical result had been many times greater.

9. Having satisfied myself, in a variety of ways, of the extreme delicacy and promptitude of action of this instrument, I thought of applying it to detect the heat of the moon's rays in a more unexceptionable manner than, I am persuaded, it has ever been attempted. This curious question had not escaped MM. Nobili and Melloni when they first constructed the instrument, and they mention in their first account of the thermo-multiplier their attempts at its solution*. But, like previous experimenters, they employed a metallic mirror to concentrate the rays of the moon, which, acting in the usual manner of dispersing the heat of the thermometer, produced sogreat a cooling effect, as completely to neutralize any positive results.

10. It occurred to me, however, from the consideration of M. Melloni's very decisive experiments as to the permeability of screens of different kinds to heat from various sources, that the moon's heat must, in very great proportion at least, radiate through glass. And this on several grounds; as, 1 . because the sun's heat, of which this may be considered as an integral part, does so with scarcely any loss; 2 . because heat, accompanied by light, always does so, and generally in proportion to the brilliancy and refrangibility of that light; and, 3. because the lunar rays having passed through the whole thickness of the atmosphere must, according to the experiments of De la Roche, fully confirmed by Melloni, have parted with the greater part of that species of heat most easily stopped, and hence arrive at the earth in a state comparatively capable of passing through glass and similar substances. If this opinion be correct (nor can I entertain any doubt upon it), if we substitute a lens for a mirror to concentrate the lunar rays, we shall profit by all, or nearly all, of their heating effect, whilst such a lens, instead of promoting the radiation of the heat of the thermometer to the sky, will entirely stop it (because heat of this description does not pass sensibly through the thinnest glass), and thus its disturbing influence will be entirely prevented.

11. I employed, therefore, a polyzonal lens made by Soleil of Paris, in my custody, to concentrate the moon's light. The diameter of the lens is 30 inches; its focal distance about 41

* Annalcs de Chimie et de Physique, December 1831. 
inches, whence we may compute the size of the lunar image to be a circle 0.38 inch in diameter. Comparing this with the dimensions of the intercepted cylinder of rays, we shall find the concentration to exceed 6000 times. But even if we admit that half the rays are reflected, dispersed and absorbed, we shall have still an effective increase of 3000 times.

12. My experiments were made on the 16 th December 183 , between 9 and 11 o'clock, the moon being only 18 hours past full, and (towards the close) less than 2 hours from the meridian. She was also particularly high, having a declination of $25^{\circ}$ north. The thermal pile, which was particularly commodious for the experiment, had one extremity elevated to the proper angle, and being placed accurately in the focus of the mirror, the moon's image was brilliantly thrown on the extremity of the pile. The sky was on the whole very pure, though an occasional milkiness was perceived, but the best observations were made at the clearest moments, because then the air was also most still; for though the instrument was placed in a most sheltered spot, the faintest breeze was indicated by a deflection of the needle, and with such promptitude, that I generally could perceive in this way its approach before I could feel it. The action of the lens was so perfect, that the image was perfectly sharp, and the spots clearly defined. The lunar rays were alternately screened and admitted by an assistant passing a sheet of pasteboard across the surface of the lens next the moon; for when it was interposed between the lens and the instrument, a sensible disturbance took place. By these and other precautions, the needle was steady beyond my expectations, and during an hour and a quarter that the observation lasted, I had probably at least twenty perfectly unexceptionable comparative observations, free from the influence of wind, and which invariably gave not the faintest indication of warmth. When I got a deviation of the needle at the moment of unscreening the moon's rays, I verified it by screening them instantly, and watching for a return to zero, but I was always disappointed. I feel quite confident that the effect, if there was any, could not amount to a quarter of a degree of the galvanometer ; and, owing to the dynamical effect which I have described of a first impulse, that it is improbable that it amounted to half that quantity.

13. Hence it becomes an object of interest to form some estimate of the sensibility of the thermo-multiplier, compared to common thermometers. It would be difficult to give a precise measure of the degrees of temperature of the two extremities of the pile*, but we may compare the effect of equal

* This might best be done by adapting a differential thermometer of extreme delicacy, so that the balls might be in contact with the two extreT 2 


\section{Prof. Forbes on the Refraction and Polarization of Heat.}

quantities of heat upon this and another instrument. For this purpose I employed two air thermometers of great delicacy ; one was the photometer of Leslie, having one ball covered with lamp black, and exposed to the same source of heat as the pile, whilst the other ball was shaded. The other instrument was a vertical differential thermometer, having a hemispherical reflector, intercepting a cone of rays 2.50 square inches in section. I found it impossible to operate with small degrees of heat, which could not be reckoned accurately on the air thermometers, owing to their tardy action; but, from several experiments, I concluded that the same quantity of heat falling on the photometer ball and on the pile, moved the liquid of the former through $1^{\circ}$, and the needle of the multiplier through 4.2 . The degrees of the photometer being loths of $1^{\circ}$ cent., one centigrade degree would correspond to $42^{\circ}$ of the galvanometer (assumed of equal value throughout the scale). The experiment with the differential thermometer, being similarly conducted, gave for the effects of equal quantities of heat, $1^{\circ}$ cent. to $62^{\circ}$ of the multiplier. If we assume from these experiments that a quantity of heat which raises an air thermometer by one fiftieth of a centigrade degree, affects the galvanometer by $1^{\circ}$, since a quarter of a degree of the latter is a measurable quantity, and half of that may be estimated as a sensible impression, we may measure an effect of $\frac{1}{2} 0$ of a centigrade degree, and perceive (by unassisted vision), an effect of $\frac{1}{40} \overline{0}$.

14. In the case of the moon's rays, concentrated 2000 times, we have seen that it is improbable that even the last effect was produced. The whole sensitive extremity of the pile being larger than the moon's image, was not brought into action; but if we compare their relative dimensions*, we shall still find that it is improbable that the direct light of the moon would raise a thermometer one three-hundred-thousandth part of a centigrade degree, at least in this climate.

15. The value of the thermo-multiplier consists not so much in the minuteness of its indications, which may easily be equalled by employing large enough thermometers, but in the certainty and rapidity of its action. Air thermometers, such as I compared it with, though the size of the balls was inconsiderable, required so long a time to assume their temperature,

mities of the pile, and the spaces round them filled up with copper filings, or some such material. But the experiment could bardly be quite decisive.

* The moon's image contained 0.114 square inches, whilst the area of the pile is about $0 \cdot 40$. Hence little more than a fourth of the pile was brought fully into action; but any dispersed light (for which we have made allowance), would act on the neighbouring parts. 
that, when exposed simultaneously with the thermal pile to the source of heat, the latter had almost assumed its maximum effect before the others had sensibly moved; and it is obvious that, in delicate experiments, where constancy in the producing cause is presumed, rapidity of execution is essential. In short, with an air thermometer (which requires from 10 to 15 minutes to give a single result), the greater part of the experiments to be described would have been impossible from this cause alone, and the remainder would have been tedious beyond measure. It will therefore be conceived that were thermometers enlarged so as to give as minute indications as the multiplier, they would be utterly unmanageable.

16. Of all the researches of $M$. Melloni on radiant heat that of the refrangibility of non-luminous heat by a prism of rock salt is the most striking. Viewing it in connexion with the theory of heat, and its analogies with light, this experiment is even more important than those connected with the very obscure subject of absorption, which has been illustrated by his numerous deterrninations of the stoppage of radiant heat, by screens or media of different kinds. At the time when I commenced these experiments, in November last, I was not aware that M. Melloni had published a second memoir, which, after many of my experiments were made, I met with in the fifty-fifth volume of the Annales de Chimie. It appeared to me a matter of great interest to determine the refrangibility of non-luminous heat by direct experiment; and, in doing this, I was led to verify, in the fullest manner, the published experiments of $\mathrm{M}$. Melloni on the refraction of heat, not merely derived from brass heated by an alcohol lamp, so as not to have the faintest luminosity in the dark, but also of heat derived simply from water under its boiling point. I found that so admirable was the sensibility of the instrument, that we may determine, with great accuracy, by repeated trials, the angular position of the prism which gives the maximum effect; and, having given the angles made by the incident and emergent rays with the sides of the prism under those circumstances, we may compute the index of refraction for the rock-salt, in regard to rays of heat. Upon making the calculation, it appeared that the direction thus experimentally found, gave nearly the same result as for light, which was an ample proof of the reality and striking nature of the experimental result; but it at the same time appeared that the whole dispersion for the spectrum is so inconsiderable, that, in this way, we could hardly expect to obtain a numerical result for the dispersion of the heating rays. I afterwards found, upon reading $M$. Melloni's second memoir, that he had experienced the same difficulties, and that, though he constructed a pile on purnose, 
he had not succeeded in obtaining numerical results. He found, bowever, that the refrangibility of the rays diminished with their temperature. I also obtained a slight refraction of non-luminous heat through a glass prism.

17. But if heat be capable of refraction by the ordinary agents, an important question arises, Is the phænomenon of double refraction common to heat and light? Rock-salt, the only substance yet discovered which transmits dark heat in large quantity, does not possess this power. To attempt it with Iceland spar would certainly be fruitless, from the very small transmitting power which it possesses, besides some other practical difficulties which suggest themselves. It must be by more refined processes that we can detect this property. Such will be stated in the sequel.

[To be continued.]

\section{Proceedings of Learned Societies.}

ROYAL SOCIETY.

1834. A PAPER was read, entitled, "On the Determination Nov.20.- A of the 'Terms in the disturbing Function of the fourth Order, as regards the Eccentricities and Inclinations which give rise to secular inequalities." By J. W. Lubbock, Esq., V.P. and Treas. R.S.

The author observes, that the magnitude of the terms of the fourth order in the disturbing function, relating to the inclinations, in the theory of the secular inequalities of the planets, does not admit of being estimated a priori; and consequently the amount of error which may arise from neglecting them cannot be appreciated. The object of the present investigation is to ascertain the analytical expressions of these terms; and the method adopted for this purpose is derived from principles already explained by the author in a former paper. He has bestowed great pains in putting these expressions into the simplest form of which they are susceptible; and has finally succeeded, after much labour of reduction, in obtaining expressions of remarkable simplicity. He exemplifies their application by the calculation, on this method, of one of the terms given by Professor Airy as requisite for the determination of the inequality of Venus; and arrives, by this shorter process, at the same result. The same method, he remarks, is, with certain modifications, applicable to the development of the disturbing function in terms of the true longitude.

A paper was also read, entitled " Note on the Astronomical Refractions.” By James Ivory, Esq., K.H., M.A., F.R.S.

The object of this communication is to show how far the author has been successful in establishing the true theory of astronomical refractions, in his paper published in the Philosophical Transactions for 1823 , by comparing the results of that theory with the best and most recent observations; namely, those recorded in the "Funda- 